Research Report 1772

\title{
Assessing and Managing User-Produced Training Support Packages
}

\author{
J. R. Gossman and Christopher R. Graves \\ Human Resources Research Organization \\ Rebecca P. Mauzy \\ L-3 Communications \\ Robert A. Clagg \\ Litton PRC
}

\section{Armored Forces Research Unit \\ Barbara A. Black, Chief}

U.S. Army Research Institute for the Behavioral and Social Sciences 5001 Eisenhower Avenue, Alexandria, Virginia 22333-5600

May 2001

Army Project Number 20363007A792
Personnel Performance and Training

Approved for public release; distribution is unlimited. 